\section{NOTAS SOBRE LA CRISIS DE CUIDADOS: DISTRIBUCIÓN SOCIAL, MORALIZACIÓN DEL TIEMPO Y RECIPROCIDAD DEL TIEMPO DONADO EN EL ÁMBITO DOMÉSTICO-FAMILIAR}

\author{
Matxalen Legarreta Iza \\ Universidad del País Vasco / Euskal Herriko Unibertsitatea \\ matxalen.legarreta@ehu.eus \\ ORCID iD: http://orcid.org/0000-0001-7892-325X
}

Cómo citar este artículo/Citation: Legarreta Iza, M. (2017). Notas sobre la crisis de cuidados: distribución social, moralización del tiempo y reciprocidad del tiempo donado en el ámbito doméstico-familiar. Arbor, 193 (784): a381. doi: http:// dx.doi.org/10.3989/arbor.2017.784n2004

Recibido: 17 junio 2014. Aceptado: 09 mayo 2016.

RESUMEN: Los cambios acaecidos en las sociedades occidentales en las últimas décadas configuran una nueva forma de responder a la provisión de cuidados que ha sido definida en términos de crisis. Esta nueva organización agudiza las desigualdades de género existentes y genera nuevas relaciones de poder asimétricas aunque, al mismo tiempo, ofrece también oportunidades de resignificación del trabajo doméstico y los cuidados. El artículo analiza la crisis de cuidados en relación a la distribución social del tiempo donado en el ámbito domésticofamiliar, así como a su significación y representaciones (moralización y reciprocidad). Para ello, emplea los datos cuantitativos producidos mediante la Encuesta de Presupuestos de Tiempo del Instituto Vasco de Estadística - Eustat y el material generado a través de grupos de discusión.

PALABRAS CLAVE: Crisis de cuidados; tiempo donado; reciprocidad; distribución social del tiempo; moralización del tiempo; adscripciones de género.
SOME NOTES ON CARE CRISIS: SOCIAL DISTRIBUTION, TIME'S MORALIZATION AND RECIPROCITY OF TIME DONATED IN THE DOMESTICFAMILIAR DOMAIN

Copyright: (C) 2017 CSIC. Este es un artículo de acceso abierto distribuido bajo los términos de la licencia Creative Commons Attribution (CC BY) España 3.0.

ABSTRACT: The changes in Western societies in recent decades constitute a new way of responding to the provision of care that has been defined in terms of crisis. This new organization exacerbates existing gender inequalities and generates new asymmetrical power relations while, at the same time, it also offers opportunities for redefinition of domestic work and care. The article analyzes care crisis in relation to the social distribution of donated time in the domestic-family domain, as well as its significance and representations (moralization and reciprocity). For this purpose, it uses the quantitative data produced by Time Use Budget's Survey of the Basque Statistics Institute-Eustat and material generated through focus groups.

KEYWORDS: Care crisis; donated time; reciprocity; time's social distribution; moralization of time; gender ascriptions. 


\section{INTRODUCCIÓN ${ }^{1}$}

La crisis de cuidados hace referencia a los desajustes en el sistema de provisión de cuidados que surgen en las últimas décadas en los países occidentales a raíz de las transformaciones en las estructuras y en los modos de vida y sus significados y representaciones. Principalmente, como consecuencia del aumento de la esperanza de vida, crece el número de personas que precisan cuidados y, dado el debilitamiento de los estados de bienestar, son las familias (y en ellas, sobre todo, las mujeres) las que deben hacer frente a su provisión. La crisis de cuidados es, asimismo, una crisis global que agudiza las desigualdades de género existentes, al tiempo que crea nuevas relaciones de poder asimétricas entre mujeres y hombres y entre unas mujeres y otras. Como toda crisis, sin embargo, ofrece también oportunidades para la resignificación, en este caso, de las relaciones de género, concretamente, con relación al trabajo doméstico y los cuidados.

El presente artículo pretende ofrecer una reflexión sobre la crisis de cuidados a través del estudio del tiempo donado en el ámbito doméstico-familiar ${ }^{2}$. Concretamente, el objetivo es analizar cómo se responde a la provisión de cuidados atendiendo tanto a la distribución y el empleo del tiempo de trabajo doméstico-familiar, como a su significación y representaciones (moralización y reciprocidad). Para ello, se han empleado los datos cuantitativos producidos por el Instituto Vasco de Estadística - Eustat a través de la Encuesta de Presupuestos de Tiempo (en adelante, EPT) ${ }^{3}$ y el material cualitativo generado mediante grupos de discusión (GD) en el marco de una investigación llevada a cabo por la autora ${ }^{4}$.

Además de la introducción, el artículo se divide en cinco apartados. El primero ofrece un breve esbozo sobre la crisis de cuidados y sus principales características. El segundo da cuenta de las herramientas analíticas que se emplearán para el análisis: la naturaleza del tiempo donado y los atributos del principio de reciprocidad. El tercero recoge el estudio de la distribución social del tiempo de trabajo doméstico y de cuidados tomando como base las variables socio-demográficas básicas (género, edad y relación con la del mercado laboral), y el cuarto, el de sus significados y representaciones respecto a cuatro posiciones discursivas (identificación, problematización, reinvención y reafirmación). Por último, el quinto muestra las conclusiones.

\section{CRISIS DE CUIDADOS}

Los cambios acaecidos en las sociedades occidentales contemporáneas, tanto en el nivel estructural como en el sentido de los significados y las representaciones sociales, han generado transformaciones en el modelo de organización social y, más concretamente, en las formas de provisión de cuidados. Esta situación ha sido definida en términos de crisis de cuidados por diferentes autoras (Bimbi, 1991; Ehrenreich y Hochschild, 2002; Hoschschild, 1995; Orozco, 2011; Paperman, 2011; VV.AA., 2004 y Zimmerman, 2006, entre otras).

Con el aumento de la esperanza de vida y el envejecimiento de la población, crece el volumen de población mayor y, con ello, el número de personas que precisan cuidados. En este contexto, a falta de un fortalecimiento de los estados de bienestar, son las familias ( $y$, en ellas, principalmente, las mujeres) las que tienen que hacerse cargo de la cobertura de los mismos (véase García Sainz, en este mismo volumen). Paralelamente, la creciente participación de las mujeres en el mercado laboral y las transformaciones en las unidades de convivencia y en las formas de empleo (Bettio, Simonazzi y Villa, 2006), conllevan que en los entornos doméstico-familiares la provisión de cuidados ya no se asuma por completo en los mismos términos que antaño.

La nueva organización del cuidado se configura, además, en el nivel internacional, generando situaciones complejas de "múltiples crisis de cuidados" (Zimmerman, 2006) y "cadenas globales de cuidado y asistencia" entre mujeres de diferentes lugares del mundo (Hochschild, 2001), pues el "déficit de cuidado" (Ehrenreich y Hochschild, 2002, p. 8) de los países del norte está siendo suplido, en cierta medida, por la labor de personas migrantes que proceden de los países del sur. Todo ello conlleva que, en muchos casos, se agudicen las desigualdades existentes entre géneros, al tiempo que emergen nuevas formas de desigualdad por razones de clase, etnia o lugar de procedencia, tanto entre mujeres y hombres, como entre unas mujeres y $\operatorname{otras}^{5}$ (Anderson, 2000; Díaz Gorfinkiel, 2008; Parella Rubio, 2003; Salazar Parreñas, 2001). La crisis, no obstante, genera también oportunidades de resignificación del trabajo doméstico-familiar y de las relaciones de género sobre las que se desarrolla.

Desde una mirada macro, se afirma que la crisis de cuidados no hace más que reflejar una crisis más profunda que supone el cuestionamiento de una forma de estructurar la sociedad y entender el mundo en el que vivimos, desarrollada y afianzada en los países occidentales a partir de la emergencia del capitalismo industrial (Orozco, 2010; Orozco, 2011). Desde una mirada micro, la crisis se refleja en los cambios 
en las dinámicas de los entornos doméstico-familiares, principalmente en lo referente a los discursos y representaciones en torno al sistema de provisión de cuidados. El artículo se centra en la mirada micro para ofrecer un análisis del tiempo donado en el ámbito doméstico-familiar.

\section{DON, RECIPROCIDAD Y MORALIZACIÓN DEL TIEMPO}

Hablar de tiempo donado no es novedoso. Tal como queda reflejado en reflexiones anteriores de la autora (Legarreta, 2012), es posible hallar referencias a este término en otros trabajos anteriores ${ }^{6}$. Barbara Adam, por ejemplo, señala que "las relaciones sociales están permeadas por la donación de tiempo" (Adam, 1999, p. 10). De esta forma, alude a una concepción del tiempo que no se corresponde con el "tiempo vacío" que se compra y se vende en el mercado ("tiempo de reloj"), y a una noción de lo social que no se rige únicamente por criterios utilitaristas. Ramón Ramos, por su parte, aborda la donación de tiempo en relación al trabajo doméstico-familiar en varios de sus textos sobre las metáforas de tiempo de la vida cotidiana (Ramos Torre, 2007; Ramos Torre, 2009; Ramos Torre, 2011). Según el autor, el tiempo empleado en este ámbito se define por su carácter moral, así como por su densa carga emocional: "sería reductivo pretender que (...) [el tiempo] sólo puede ser un bien económico sometido a cálculo y valorable según criterios de utilidad material (Ramos Torre, 2007, p. 180).

Ciertamente ambas propuestas coinciden al hacer referencia al tiempo como elemento constitutivo de una forma específica de relación: el don. En ambos casos el tiempo es algo que se dona. Así, al hablar de tiempo donado entran en juego al menos dos aspectos que merecen ser abordados con mayor exhaustividad: por un lado, la naturaleza de la relación que se crea en torno al tiempo y, otro lado, la naturaleza del tiempo en tanto que objeto de la relación. En cuanto a la naturaleza de la relación, el don hace referencia a una relación basada en la reciprocidad. En cuanto a la naturaleza del tiempo, la moralización es un atributo inherente del tiempo-don. A continuación, se desarrollan estos dos aspectos con mayor precisión.

En primer lugar, respecto al don en tanto que relación basada en el principio de reciprocidad, se ha tomado como referencia principalmente la obra clásica de Marcel Mauss (1925/1971) ${ }^{7}$. El antropólogo francés describe el don como una relación de intercambio que se desarrolla ajena a la lógica utilitarista del mercado y que conlleva tres acciones: dar, recibir y devolver. La contraprestación forma parte de la relación, por lo que el don, aparentemente altruista, supone interés: la persona que ofrece algo espera ser compensada por ello. Cuando alguien da, quien recibe contrae una deuda con el donante, de forma que el don comporta aceptación y retorno. La reciprocidad, por tanto, es una característica constitutiva del don. Así, en base a unas pautas que permanecen tácitas y que son aceptadas por las partes implicadas en la relación (Casado Neira, 2003), el don genera una forma de relación que conlleva, paralelamente, como fundamento y resultado, la creación de vínculos. Siguiendo a Joan Bestard se puede afirmar que "el don que no intensifica la solidaridad social es una contradicción" (1998, p. 224). En la obra de Mauss el don se presenta como el fundamento mismo de la sociedad.

En esta misma línea, Alvin W. Gouldner define como universal el principio de reciprocidad, matizando que la norma moral de la reciprocidad interiorizada por las partes que conforman la relación "es un mecanismo concreto y específico involucrado en el mantenimiento de todo sistema social estable", pues obliga moralmente a dar beneficios a aquellos de quienes previamente se han recibido (Gouldner, 1979 p. 237). No obstante, advierte que las relaciones de poder sobre las que se desarrolla la vida social, así como la falta de garantías de que se restituya lo otorgado, puede, en ocasiones, llevar a una desestabilización del equilibrio, así como a un cuestionamiento mismo del principio de reciprocidad. Se supera, de esta forma, el determinismo y la inclinación a favor del equilibrio del pensamiento funcionalista, revisado por el autor, para ofrecer un abanico más amplio de posibilidades en torno al desarrollo de la reciprocidad.

En segundo lugar, respecto al tiempo como objeto de la relación, se toma como referencia, entre otros, el trabajo de Ramón Ramos (Ramos Torre, 2007; Ramos Torre, 2009; Ramos Torre, 2011) en torno a las metáforas del tiempo. Siguiendo la propuesta de las metáforas, en este caso, el tiempo es entendido como un tiempo-recurso: un tiempo que puede ser objeto de acciones dispositivas como vender, regalar, prestar, compartir o donar. Cuando se hace referencia al tiempo donado se entiende que el tiempo es lo que se dona: lo que se da, recibe o devuelve. El tiempo es el don, pues Marcel Mauss emplea el don indistintamente para apelar tanto a la "cosa o servicio" (Mauss, $1925 / 1971$, p. 228) que se da, recibe o devuelve, como a la relación de reciprocidad que se genera en torno a ella. De este modo, en tanto que objeto de una interacción basada en el principio de reciprocidad, el tiempo posee unas características particulares 
que obligan a las partes a participar en la relación: "¿[q]ué fuerza tiene la cosa que se da que obliga al donatario a devolverla?" (Mauss, 1925/1971, p. 157).

Se trata, por tanto, de un tiempo que obliga, un tiempo que, si bien es susceptible de cuantificación, su significación no se agota en su dimensión material, sino que posee también otro tipo de cualidades: morales, relacionales y emocionales. Como apunta Laura Bimbi (1991), las obligaciones recíprocas no pueden ser medidas con la duración temporal concreta de las prestaciones ofrecidas o restituidas, por lo que el tiempo asume en el don una connotación cualitativa. Asimismo, el don implica a quien lo ofrece en su totalidad: conlleva la "entrega absoluta de la persona" (Casado Neira, 2003, p. 112), de forma que el tiempo que se dona se torna inseparable de la persona misma que forma parte de la interacción, lo que refuerza el carácter moral de la contraprestación y la dramatización pública de la donación (Mauss, 1925/1971).

En base a estas premisas, en los siguientes apartados se ofrece una reflexión sobre la crisis de cuidados en torno al tiempo donado en el ámbito doméstico-familiar, bien atendiendo a su materialidad (a través de los datos cuantitativos en torno a su distribución social), bien atendiendo a su significación moral (a través de los datos cualitativos sobre los discursos y experiencias). Tal distinción, sin embargo, se tomará con precaución, puesto que, como se ha señalado en otros trabajos, poner el acento en el contenido relacional del trabajo doméstico y los cuidados puede llevar a excluir las actividades más instrumentales (cocinar, limpiar...), así como las desigualdades de género que caracterizan su ejecución (Esquivel, 2009). De esta forma, en el primer caso, se realizará el análisis de los datos sobre el empleo y la distribución del tiempo en relación a las desigualdades de género y, en el segundo, se estudiarán los significados y las representaciones sociales tomando como eje el principio de reciprocidad.

\section{TIEMPO DONADO: DISTRIBUCIÓN SOCIAL Y DESIGUALDADES DE GÉNERO}

Los datos sobre el empleo del tiempo reflejan que la mitad de los bienes y servicios necesarios para la pervivencia de la sociedad vasca provienen del ámbito doméstico-familiar. Las mujeres desempeñan la mayor parte de dicho trabajo (el $72 \%$ ), mientras que los hombres cuentan con mayor protagonismo en la esfera productiva-mercantil (llevan a cabo el 59\% del trabajo remunerado). El tiempo de trabajo doméstico y de cuidados se distribuye de forma desigual entre mujeres y hombres: ellas dedican a dichas ocupaciones más del doble de tiempo que ellos (las mujeres 4 horas y 1 minuto y los hombres 1 hora y 38 minutos). Es más, considerando tanto el tiempo del ámbito doméstico-familiar como el del mercado laboral, las mujeres trabajan casi una hora y cuarto más al día que los hombres ( 1 hora y 12 minutos), disponiendo, en consecuencia, de una hora menos de tiempo de ocio y de vida social ${ }^{9}$, lo que incide en un reparto desigual de oportunidades sociales y personales (Murillo de la Vega, 1996), al tiempo que merma el bienestar de las mujeres.

Si observamos el tiempo empleado por hombres y mujeres en el conjunto de actividades (gráfico 1), se constata que la brecha entre el comportamiento de unos y otras es más pronunciada en lo referente al trabajo doméstico y los cuidados que en relación al resto de actividades. Cabe afirmar, por tanto, que las desigualdades de género más acusadas tienen lugar en el ámbito doméstico-familiar, por lo que poner el foco de atención en esta esfera no resulta trivial, sino que implica detenerse en uno de los núcleos duros de las desigualdades.

Los datos de la EPT ponen de manifiesto, asimismo, que el volumen de tiempo que mujeres y hombres dedican al ámbito doméstico-familiar está condicionado tanto por la edad como por la participación en el mercado laboral aunque, independientemente de su momento vital y de su posición social, es el género el que determina las pautas de la distribución del tiempo de trabajo doméstico y de cuidados.

Con relación al primer aspecto, cabe apuntar que el empleo del tiempo en el trabajo doméstico y los cuidados se incrementa según aumenta la edad, principalmente una vez entrada la población en la edad adulta: el tiempo dedicado por los mayores de 35 años dobla el empleado por los más jóvenes. La desigual dedicación entre mujeres y hombres se mantiene, sin embargo, en todos los tramos de edad, pues el tiempo invertido por ellas es siempre significativamente mayor que el dedicado por ellos, incluso entre la población más joven que ha sido socializada en un contexto de mayor igualdad formal. Los hombres más jóvenes dedican la mitad de tiempo que las mujeres de su misma edad: alrededor de una hora ellos y algo más de dos horas ellas. No obstante, la distancia entre el tiempo invertido por unas y por otros crece conforme va aumentando la edad: se puede observar una diferencia de casi tres horas en el tiempo empleado por mujeres y hombres tanto de edad adulta como mayores. Las mujeres adultas dedican 2 horas y 45 minutos más que los hombres adultos al trabajo doméstico y los cuidados y, entre la población mayor, 2 horas y 50 minutos más ellas que ellos (ver gráfico 2 ). 
Gráfico 1. Distribución del tiempo diario de mujeres y hombres (hh:mm). Comunidad Autónoma del País Vasco, 2008.

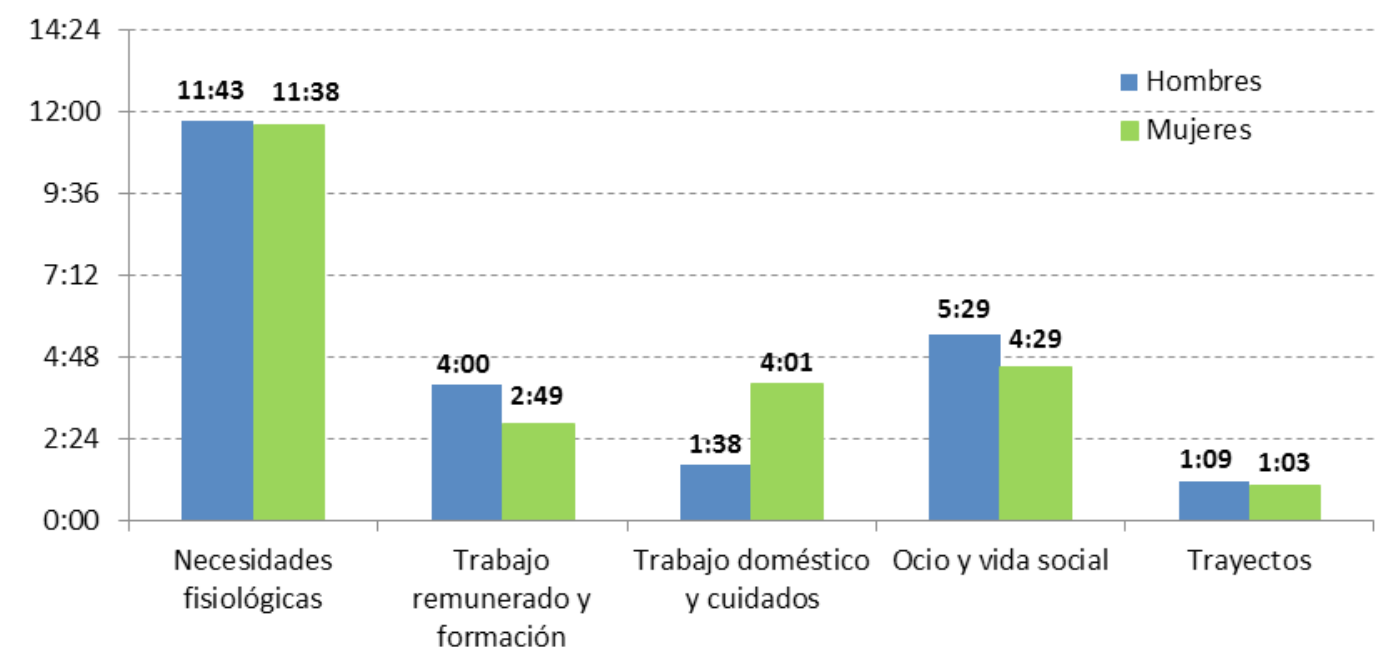

Fuente: elaboración propia a partir de los datos de la Encuesta de Presupuestos de Tiempo del Instituto Vasco de Estadística - Eustat, 2008.

Gráfico 2. Distribución del tiempo de trabajo doméstico y de cuidados entre mujeres y hombres por grupos de edad (hh:mm). Comunidad Autónoma del País Vasco, 2008.

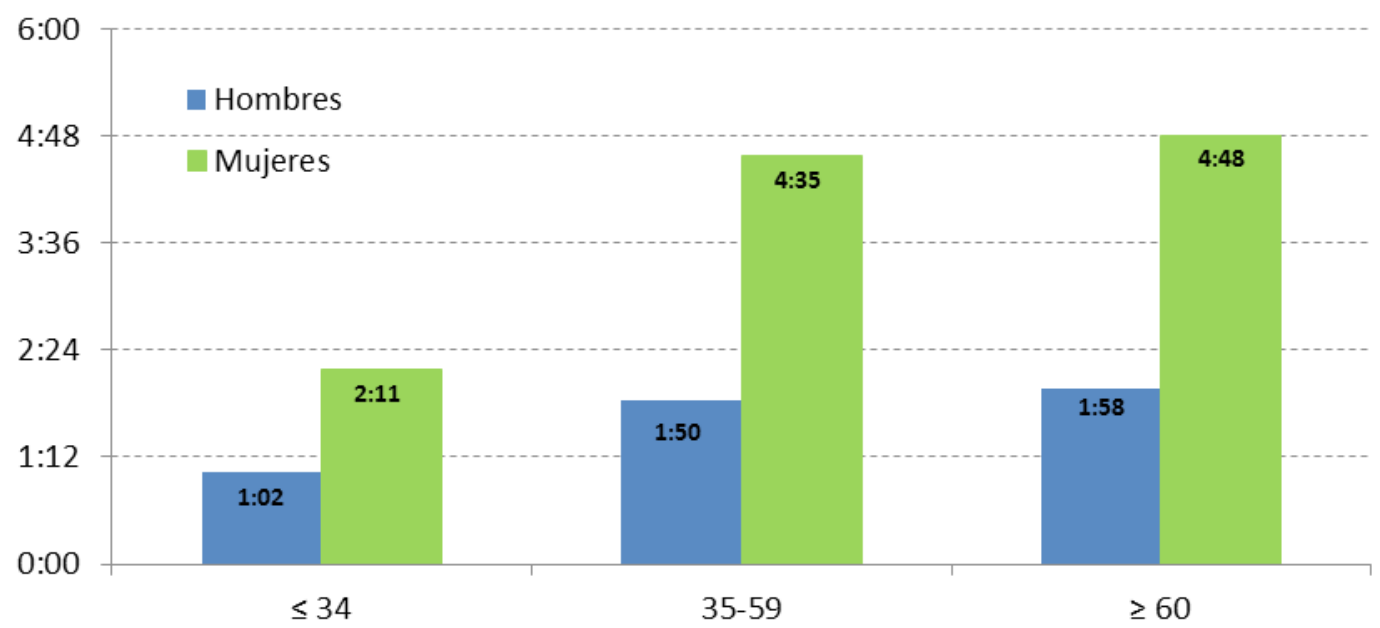

Fuente: Elaboración propia a partir de los datos de la Encuesta de Presupuestos de Tiempo del Instituto Vasco de Estadística - Eustat, 2008.

En relación al segundo aspecto, analizar los resultados según la participación en el mercado laboral aporta aún mayor número de matices a la distribución del tiempo de trabajo doméstico y de cuidados entre mujeres y hombres. Las desigualdades se mantienen en todos los casos, al margen de la relación con el mercado laboral: la cantidad de tiempo dedicado por los hombres es menor que la invertida por las mujeres, excepto entre las mujeres que son estudiantes y los hombres que no lo son. No obstante, las mujeres estudiantes emplean el doble de tiempo que sus compañeros varones en este ámbito: algo menos que una hora ellas y prácticamente media hora ellos (ver gráfico 3).

Después de la población estudiante, la empleada es la que menor cantidad de tiempo invierte en esta esfera, aunque al trabajar también en el mercado laboral, asume una carga global de trabajo considerable: casi ocho horas al día (7 horas y 42 minutos). La mayor carga recae sobre las mujeres empleadas 
que dedican más de tres horas al trabajo doméstico y los cuidados y casi cinco al remunerado ( 3 horas y 35 minutos y 4 horas y 53 minutos respectivamente). Consecuentemente, hacen frente a una carga global de trabajo de más de 8 horas y media al día ( 8 horas y 38 minutos), lo que supone una jornada semanal de más 60 horas (60 horas y 26 minutos). Se puede constatar, por tanto, que la doble jornada caracteriza el día a día de las mujeres vascas con empleo. La carga global de los hombres asalariados es menor que la de las mujeres, pese a ello asciende a 7 horas y 12 minutos.

La diferencia entre mujeres y hombres se acentúa al comparar las pautas de comportamiento de unos y otras con relación a la población desempleada y jubilada. El tiempo que dedican las mujeres en ambos casos aumenta de forma significativa, hasta llegar a igualar, en el caso de las mujeres en situación de desempleo, el tiempo que dedican a este ámbito las amas de casa a tiempo completo: casi cinco horas y media en ambos casos (ver gráfico 3). Las mujeres jubiladas emplean en el trabajo doméstico y los cuidados una hora menos que las desempleadas y las amas de casa a tiempo completo, 4 horas y 18 minutos, una cantidad sin embargo nada desdeñable. De esta forma, las mujeres en situación de desempleo y las amas de casa a tiempo completo implican en el trabajo doméstico y los cuidados la misma cantidad de tiempo que la población empleada en el mercado laboral; sin embargo, son consideradas inactivas por parte de las estadísticas oficiales sobre trabajo (la Encuesta de Población Activa). La valoración desigual de uno y otro trabajo no hace más que acentuar la relación asimétrica entre mujeres y hombres, pues no todos los tiempos tienen el mismo reconocimiento social.

Entre las mujeres desempleadas, se observa que la presencia de aquellas que desempeñan trabajos de cuidado es significativa, pues cuatro de cada diez dedica algún tiempo a esta ocupación (el 41,9\% de las mujeres desempleadas frente al $26,1 \%$ del total de mujeres), de lo que se puede deducir la existencia de una posible relación entre la maternidad y cierto alejamiento del mercado laboral. En esta línea, otros trabajos apuntan que la presencia de criaturas en los hogares conlleva un aumento del tiempo de trabajo doméstico-familiar por parte de las mujeres que corresponde con un aumento del tiempo dedicado al mercado laboral por parte de los hombres (Bacigalupe de la Hera y Martín Roncero, 2007, p. 121). Paralelamente, entre los hombres son los desempleados los que mayor volumen de tiempo dedican a la esfera doméstica-familiar, aunque invierten en ella 1 hora y 5 minutos menos que las mujeres con empleo. Se puede constatar, por tanto, que el género determina la implicación en el ámbito domésticofamiliar en mayor medida que la participación en el mercado laboral.

El gráfico 3 muestra los datos sobre la distribución del tiempo de trabajo doméstico y de cuidados de mujeres y hombres con relación a la participación en el mercado laboral.

Con todo ello, el análisis de los datos cuantitativos sobre el empleo del tiempo saca a la luz la persistencia de profundas desigualdades de género en el

Gráfico 3. Distribución del tiempo de trabajo doméstico y de cuidados de mujeres y hombres según la participación en el mercado laboral (hh:mm). Comunidad Autónoma del País Vasco. 2003.

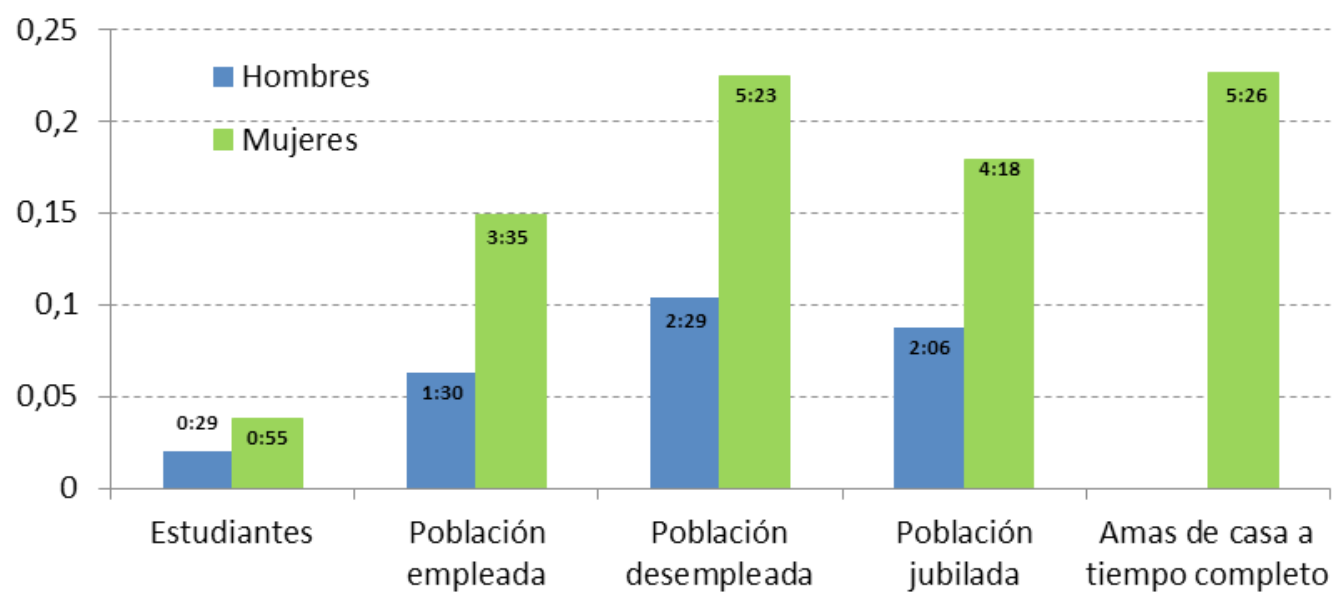

Fuente: Elaboración propia a partir de los datos de la Encuesta de Presupuestos de Tiempo del Instituto Vasco de Estadística - Eustat, 2008. 
ámbito doméstico-familiar. La participación de las mujeres en el mercado laboral implica además la asunción de una mayor carga global de trabajo por parte de ellas, lo que delimita su capacidad de acceso al tiempo de libre disposición. Así, se puede concluir que el modelo "hombre ganador de pan / mujer ama de casa" no se refleja de forma generalizada en las prácticas y en el comportamiento de la población vasca, principalmente por la participación de las mujeres en el mercado laboral. Es más, con el paso del tiempo, la Comunidad Autónoma del País Vasco muestra una tendencia que va derivando del modelo de "un trabajador y medio y una sola cuidadora" descrito por Jane Lewis (2007/2011) hacia la figura de "dos trabajadores y una sola cuidadora". Pese a ello, como se observa en el siguiente apartado, el análisis del material cualitativo deja entrever que la pauta tradicional de división sexual del trabajo sigue formando parte del imaginario social y, de forma más o menos expresa, sale a la luz en el discurso tanto de mujeres como de hombres. Al mismo tiempo, si a través de los usos y la distribución del tiempo se arroja luz sobre las desigualdades de género, una mirada en torno a los discursos y experiencias del tiempo donado en el ámbito doméstico-familiar ofrece la oportunidad de observar el desarrollo del principio de reciprocidad y sus diferentes matices. Lo pertinente no es tanto "decidir si se cumple o no la reciprocidad en términos absolutos" (Ramos Torre, 1990, p. 11), sino determinar en qué medida, en qué contextos y con qué características opera, así como en qué momentos y por qué razones deja de ser válida, se cuestiona o se rompe.

\section{TIEMPO DONADO: DISCURSOS Y EXPERIENCIAS}

A través del análisis de los datos cualitativos producidos mediante grupos de discusión, se han identificado cuatro posiciones discursivas con relación a las adscripciones de género del tiempo donado en el ámbito doméstico familiar, que vienen determinadas por las posiciones sociales concretas desde las que se producen los discursos: la identificación, la problematización, la reinvención y la reafirmación. De esta forma, como se verá en los siguientes epígrafes, las mujeres se identifican con su función doméstica, la problematizan o intentan reinventarla para apropiársela de forma renovada, y lo hacen de manera explícita. La posición de los hombres es más sutil, bien se problematiza y sale a la luz el carácter construido de la adscripción de género de la función doméstica; bien se refuerza desde el intento de desmarcarse y desvincularse de una identificación plena con el ámbito doméstico-familiar. En este sentido, la moralización del tiempo y el principio de reciprocidad se significan, se viven y se experimentan de forma diferente en unas posiciones discursivas y en otras, siendo también habitual cierta heterogeneidad en el seno de cada posición.

\subsection{Identificación: doméstico igual a mujer}

La identificación es la posición discursiva que implica la asunción de los roles tradicionales de género. Se ha identificado en relación a los discursos de las mujeres con empleo no cualificado y de las mujeres mayores. La feminización del trabajo doméstico y de los cuidados se naturaliza de forma que parece responder a la correspondencia que apunta Soledad Murillo: "doméstico es igual a mujer" (Murillo, 1995, p. 134). En relación a la moralización del tiempo, la identificación comporta un sentimiento de satisfacción por el deber cumplido.

En el caso de las mujeres mayores, su posición fuera del mercado y su socialización en los roles de género tradicionales conllevan la asunción de las funciones domésticas como parte esencial de su cotidianidad, como constitutivas de su propia identidad. Su día a día se desarrolla, en gran medida, en base a las ocupaciones doméstico-familiares, y el tiempo de cuidados se extiende más allá de las paredes del hogar y de la relación con las y los familiares más allegados, pues son habituales las visitas a parientes de diversos grados de proximidad, amistades, vecinos y vecinas... que se encuentran en residencias de mayores.

Respecto a la moralización del tiempo, se subraya el carácter altruista de la donación: ellas se ocupan y preocupan por los y las demás sin, aparentemente, esperar nada a cambio. No obstante, una mirada más atenta a sus narraciones pone de manifiesto que su dedicación es compensada por la gratitud que reciben de aquellas personas a las que prestan atención. El agradecimiento es el reconocimiento explícito del compromiso que se genera en la aceptación del don, además de un equilibrador inmediato de la deuda adquirida (Casado Neira, 2003, p. 120). El tiempo donado en las visitas a residencias es restituido de inmediato por el reconocimiento de las personas que reciben atención, en forma de contraprestación inmediata. Se cumple, por tanto, el principio de reciprocidad:

“- Pero esas cosas [se refiere a las visitas a residencias] hay que hacer, ¿̇eh? Porque agradecen.

- ¡Hombre, no!

- Fíjate tú, lo que haces. 


\section{- Y, y luego también tienes satisfacción personal \\ - También, sí, sí}

- Porque si tú ves además que se quedan contentos y que oye, pues que has estado un ratito con ellos, y aunque a poco que hayas ido, pues ellos siempre son agradecidos, sobre todo la gente que está bien de la cabeza, relativamente bien, pues lo agradecen.

-Y la que no está bien también está, la que anda ahora en la residencia se dará cuenta.

- Te agradecen todos.

- Sí, sí" (GD 8: Mujeres mayores).

Entre las mujeres con empleo no cualificado el tiempo de trabajo doméstico y de cuidados se proclama como seña de identidad, más fuerte incluso que la relación con el mercado laboral. Así, aun desarrollando un trabajo retribuido, algunas se autodefinen como "amas de casa" (Legarreta Iza, 2008, p. 62). En este sentido, se enuncian expresiones que, frente a la "rutina y sin sentido" (Arpal, 1997) del trabajo asalariado, proclaman lo doméstico-familiar como fuente de significación:

\footnotetext{
“- (...) yo en mi casa me siento superautorrealizada, yo me autorrealizo con mi vida familiar, con mi marido y... Yo si... si yo dependería de mi trabajo... (se ríe)" (GD 4: Mujeres con empleo no cualificado).
}

La moralización del tiempo se dilata en su discurso hasta llegar a tocar aspectos inherentes de la condición humana: el tiempo encarnado (Ramos Torre, 2007; Ramos Torre, 2011), tiempo del cuerpo (Adam, 1995; Menzies, 2000). En este grupo se percibe una ruptura generacional. Las más mayores apelan al egoísmo de las generaciones más jóvenes (principalmente de las mujeres) por su retraso en la edad de tener la primera criatura. Las más jóvenes no tienen hijos/as y aluden al sentimiento de culpa que les produciría tenerlos y no poder atenderlos. Las de mayor edad, con hijos/ as, posicionándose desde la perspectiva de quien, efectivamente, ha cumplido con su deber, califican de egoísta el razonamiento de sus compañeras por querer eludir las normas de reciprocidad: les toca asumir su responsabilidad adulta y ofrecer algo a la sociedad, de quien hasta ahora solamente han recibido, pues como se apunta en otros trabajos, "no transmitir la vida es cortar una cadena de la que nadie es el último eslabón" (Héritier-Augé, 1985; cf. Imaz Martínez, 2009, p. 200). Es el momento oportuno, el kairos, de empezar a prestar cuidados, ya que romper la lógica del don supone quedarse con algo que no les corresponde: responde a la lógica lineal de la acumulación y no al principio circular de la reciprocidad.

\subsection{Problematización: sentimiento de culpa e incertidumbre}

La problematización es la posición discursiva que da lugar a narraciones que ponen de manifiesto la historicidad de las adscripciones de género y su naturaleza contingente en tanto que construcciones sociales. Se identifica entre las mujeres profesionales y entre los hombres jubilados y prejubilados. Aunque por distintas razones, la problematización es reflejo en ambos casos de una tensión entre sus adscripciones de género (plano normativo), su práctica cotidiana (presente) y su horizonte temporal (tanto en relación a las expectativas que tenían en el pasado respecto al presente, como a las expectativas que tienen en el presente respecto al futuro).

Las mujeres profesionales se muestran especialmente críticas respecto a las adscripciones de género y su desigual reparto de tareas, responsabilidades y funciones. Elaboran un diagnóstico crítico en torno a una situación que, bien se define de forma abstracta y global (como característica del orden social vigente), bien se expresa de forma encarnada a través de contradicciones que atraviesan su día a día, su propia experiencia, sus inquietudes, sus motivaciones y sus expectativas. El mandato de género se enfrenta a su práctica cotidiana y a su proyección profesional, poniendo en juego la propia persona, su identidad y sus capacidades.

Dicho enfrentamiento genera que el significado moral del tiempo donado en el ámbito domésticofamiliar se traduzca, en su caso, en un sentimiento de culpa generalizado. Sentimiento de culpa frente al que, en algunas ocasiones, se cede, llegando a trastocar los ritmos del propio cuerpo (Legarreta Iza, 2008; Legarreta, 2014a $)^{10} y$, en otras, sin embargo, se lucha, llegando a cuestionar y renegociar los roles tradicionales de género ${ }^{11}$ y sale a la luz su agencia, su capacidad de actuar y de empoderarse. Asimismo, su postura crítica frente a las adscripciones de género pone de manifiesto las desigualdades que genera el modelo heredado de domesticidad (Legarreta Iza, 2012) que supone una disposición de estar atenta a cubrir las necesidades de los demás y obviar las propias (Murillo de la Vega, 1996). Frente a la actitud de entrega, que implica la renuncia del principio de reciprocidad, se aboga por reivindicar la contraprestación en el marco de un modelo más equitativo de relaciones de género, una demanda que recuerda al "nuevo pacto social entre géneros" formulado por Jane Lewis (2007/2011):

“- (...) Pero en el fondo, iarg!, arrastraba todavía esa cosa, que si estás un poco baja ya te cogen la culpabilidad, ya te han agarrado la culpabilidad, y vuelves 
a regresar, y vuelves a repetir, y eso es que estamos educadas ahí a renunciar de nuestra calidad a favor de los demás (...) te van educando, te van educando para dar, y dar, y cuando recibes, que parece que tendría que ser una contrapartida, como cuando haces un balance financiero...

- Te sientes culpable.

- ...que tienes un activo y un pasivo, y tú das, entra y sale, ¿no?, y sale y entra, y tienes una compensación. Todavía, cuando recibes todavía de vez en cuando dices "gracias, gracias, gracias", ¿no?

- Uhm Porque... (silencio)

- Ya, no estás acostumbrada

- ...yo creo que tenemos que equilibrar esos balances" (GD 5: Mujeres profesionales).

En el caso de los hombres jubilados y prejubilados, la problematización se desarrolla desde un desajuste encarnado entre su socialización, el ejercicio de su rol durante su vida adulta (como "ganador de pan") y la situación en la que se encuentran actualmente. En su discurso se percibe una implicación notable con el ámbito doméstico-familiar. Este cobra una centralidad que no se observa en las narraciones del resto de los grupos compuestos por hombres: se abordan aspectos relacionados con el tiempo dedicado al cuidado específico de criaturas y de personas mayores, así como con otros quehaceres domésticos. Por ello, su experiencia vital del presente rompe con el modelo de ciclo vital lineal del discurso hegemónico: juventudestudio, edad adulta-empleo, vejez-jubilación (Cordoni, 1993; Legarreta, 2014b). Sus vivencias no coinciden con las expectativas que tenían en torno a su salida del mercado laboral, encontrándose en el presente inmersos en un ámbito, el doméstico-familiar, que les es ajeno y sobre el que experimentan una sensación de deber y de obligatoriedad.

La moralización del tiempo donado en lo doméstico-familiar se caracteriza por la queja, la preocupación, el enfado y la resignación, pero sobre todo por la incertidumbre y el desconcierto. Asumen que ellos mismos llegarán a la situación de aquellas personas mayores que, hoy en día, demandan sus cuidados. Hacerse mayor se identifica con ser más vulnerable y poseer menor grado de autonomía y, viceversa, la dependencia se iguala a la vejez:

“- ¿Cómo voy a ir yo [a la residencia] a aguantar viejos, ahí al lado? Aunque sean más jóvenes que nosotros (...)" (GD 9: Hombres jubilados o prejubilados) ${ }^{12}$.
Prevén que envejecerán y precisarán cuidados, pero ¿quién se los va a prestar? La pregunta está implícita y siembra la incertidumbre: surge la duda y la desolación, pues no se atisba ninguna respuesta clara.

Como apunta Gouldner, "la norma de la reciprocidad conduce a un interés por el pasado (...) [y] también sensibiliza a la persona con respecto al futuro" (1979, p. 247), pues la relación de reciprocidad se establece, desde la perspectiva de sus protagonistas, que actúan y dan sentido al presente, bien en relación al pasado, bien respecto a sus expectativas de futuro. En este caso, haber recibido tiempo de trabajo doméstico y cuidados en el pasado les compromete a ofrecer su tiempo en el presente y a esperar una contraprestación en el futuro. El principio de reciprocidad toma un sentido $u$ otro en relación al horizonte temporal de sus protagonistas. La narración, sin embargo, deja entrever la ruptura de la norma de reciprocidad, así como cierto resquebrajamiento de las relaciones intergeneracionales. Siguiendo a Gouldner, se pone de manifiesto, por tanto, que la reciprocidad, "aunque necesaria para la estabilidad de los sistemas sociales, no [siempre, cabría matizar] engendra requisitos morales suficientes para tal fin" (1979, p. 247).

“- Esta generación que estamos aquí somos la que peor, la que peor va a vivir, seguramente... Nos ha tocado cuidar de nuestros hijos, nos ha tocado obedecer (énfasis) a nuestros padres...

- Y cuidarlos

- Y cuidarlos...

- Sí.

- Y nuestros hijos...

- Nada.

- En plan suave, van a decir... Te van a dar la patada.

- Exacto.

- Sí, sí, te van dando largas, sí.

- Pero si no es en plan suave, si es que esto es así.

- Es que esto es así.

- O que la vida ha evolucionado así, pero que esta generación..." (GD 9: Hombres jubilados y prejubilados).

En este contexto, el quebrantamiento de las normas no hace más que poner de manifiesto su existencia misma, la de una lógica circular que rige la relación: dar, recibir y devolver. El don se constituye en tanto que sistema normativo que pauta las reglas de la provisión de bienestar y cuidados en el ámbito doméstico-familiar: quién, cuándo, cómo y con qué intensidad debe prestar cuidados o recibirlos. 


\subsection{Reinvención: "marujas con glamour"}

La reinvención es la posición discursiva que redefine la identificación de las mujeres con su función doméstica. Se desarrolla principalmente entre las mujeres amas de casa a tiempo completo. Ellas describen su situación actual como consecuencia de una elección tomada en el pasado, en un momento concreto de su trayectoria vital; elección que, con el paso del tiempo, ha resultado irrevocable. Dejan de trabajar de forma retribuida para cuidar de sus hijas e hijos, pero asumen que, aunque quisieran volver al mercado laboral, no se podrían adecuar a él, puesto que carecen de la cualificación y las prestaciones que demanda. En este sentido, admiten que su situación del presente no está generalizada entre las mujeres y desarrollan un posicionamiento en torno a su "elección" que no siempre se construye de forma monolítica.

Su discurso y su autodefinición como grupo de iguales se desarrolla guardando distancia de otros dos grupos: "madres que trabajan" y "marujas". Por un lado, desaprobando el comportamiento de las madres con empleo, reafirman su propia decisión: ellas, frente a las otras, ejercen una maternidad intensiva, de forma responsable y con plena disposición. Por otro lado, desligándose de la proyección de una imagen trasnochada (reflejada en el término "maruja") crean una identidad renovada en torno a su rol de esposas y madres: "marujas con clase, con glamour" (GD 5: Amas de casa a tiempo completo). Con esta estrategia doble pretenden construir una identidad que las sitúe en un lugar más relevante en el imaginario social y que revalorice su trabajo y su propia existencia.

Entre la autoafirmación frente a las "madres que trabajan" y la resignificación frente a las "marujas", se desarrolla un discurso que no siempre se sostiene de forma sólida, pues presenta contradicciones, fisuras, dudas y desajustes cuando se hace referencia al futuro de sus propias hijas: ¿las animarían a tomar su camino?, ¿y si ellas deciden hacerlo? No hay acuerdo sobre este aspecto, lo que evidencia el cuestionamiento de los roles tradicionales de género:

“- Yo tengo una contradicción como mujer, de que yo estoy muy bien, ¿no?, pero claro, yo no cobro un sueldo, yo soy ama de casa, yo dependo total de mi marido, en todos los aspectos. ¿Bien? Te va bien, te va bien, pero claro, ¿yo para mi hija qué quiero? Yo no quiero lo mío y estoy muy bien...

\footnotetext{
- Yo tampoco.
}

- Pero al mismo tiempo, claro, luego como mujer, me planteo, tengo una contradicción, ¿̇yo qué quiero? que, que mi hija, ella, ella tenga su carrera, ella dependa y ella diga.

(Hablan todas juntas, no se entiende).

- Que elija ella su vida, que elija su vida ella.

- Igual ella elige quedarse en casa como tú.

- No, no, no, yo jamás le aconsejaría a mi hija que se quedase en casa.

- Claro.

- ¡No, no, no!

- Jamás, jamás.

- Pero ella puede elegir.

- Sí, sí, puede elegir, pero yo...

- Yo si alguna vez tiene un hijo, si alguna vez tiene un hijo mi hija, a mí sí me gustaría que hiciese lo mismo que he hecho yo, porque desde luego, como la madre y como el padre nadie le... nadie le va a atender, ninguna persona, sea la persona que sea...

- $\quad$ Estoy de acuerdo.

- Yo no le diría que dejase su...

- No, pero no me digas, si decide quedarse igual que hemos decidido...

- Tampoco me voy a martirizar porque se quede" (GD 3: Amas de casa a tiempo completo).

En su día a día las amas de casa a tiempo completo dedican un volumen importante de tiempo al ámbito doméstico-familiar, pero su valor moral no siempre se expresa en los mismos términos. En ocasiones, se manifiesta a través de la satisfacción, principalmente cuando se contrapone a las vivencias menos intensas en torno al cuidado de la prole por parte de sus parejas o por parte de las madres con empleo. En otras, sin embargo, se deja entrever que las cosas podían haber sido de otra forma, que su elección conlleva renuncia y sacrificio. En estos últimos casos, la moralización se expresa en términos de compensación y puede ser interpretada en relación al principio de reciprocidad como un reajuste del equilibrio entre aquello a lo que se ha renunciado y lo que se ha obtenido a cambio:

“- (...) yo lo que más valoro es eso, que cuando mis hijos llegaban a casa yo estaba en casa, entonces era lo que más valoraba, el hablar con ellos, el contar, el estar en todo ahí... (énfasis) (...) pero yo creo que eso lo valoras mucho, que mis hijos, cuando he llegado a 
casa han estado, yo he estado allí, yo creo que ha sido el gran premio a... todos estos años" (GD 3: Amas de casa a tiempo completo).

\subsection{Reafirmación: hombre ganador de pan / mujer ama de casa}

La reafirmación es la posición discursiva que refuerza la división de roles del modelo "hombre ganador de pan / mujer ama de casa". Se desarrolla mayoritariamente entre los hombres adultos con empleo. Parten de una posición de colaboración y de reparto de tareas en el ámbito doméstico-familiar, pero no se percibe una asunción plena de las responsabilidades que conlleva. El trabajo doméstico y los cuidados no resultan temas centrales en el desarrollo de su narración. Salen a la luz como constitutivos de las rutinas diarias y aquellos que tienen criaturas pequeñas ponen de manifiesto los problemas de "encaje temporal" (Lewis y Weigert, 1981/1992) y la dificultad de disponer de tiempo libre y de ocio.

Los hombres con empleo dedican al trabajo doméstico y los cuidados una cantidad de tiempo considerablemente menor que la que dedican tanto los hombres jubilados como los desempleados. Además, su relato sobre este tiempo no posee la intensidad de la narración de los hombres jubilados y prejubilados, a la que se ha hecho alusión anteriormente, sino que se trata más bien de un discurso aséptico, des-encarnado, que no se construye como parte esencial de su ser. Se puede percibir, por tanto, cierto distanciamiento de las funciones domésticas. Un ejemplo de ello es la escasa resonancia que tiene en el grupo de hombres con empleo no cualificado que uno de ellos lleve un tiempo con reducción de jornada gracias a un permiso de paternidad. Si bien el tema sale a colación a través de una intervención de la moderadora, el grupo no produce interlocución, no elabora un discurso encarnado sobre este aspecto.

Así, pese a que los hombres con empleo afirmen ocuparse del trabajo doméstico y los cuidados, no se percibe por su parte una preocupación generalizada por este ámbito. Se puede constatar, por tanto, que en el nivel discursivo no reproducen modelos de corresponsabilidad, sino que reafirman la significación y el peso simbólico que sigue teniendo en el imaginario social el modelo "hombre ganador de pan / mujer ama de casa", un modelo de relaciones desigual en el que a priori la jerarquización de espacios, tiempos y ocupaciones hace difícil el establecimiento de unas pautas de interacción sustentadas sobre un principio de reciprocidad simétrica.

\section{CONCLUSIONES}

La organización social de provisión de cuidados se ha definido en términos de crisis, pues las pautas tradicionales de sostenibilidad de la vida han dejado de funcionar como antaño. Un acercamiento a las dinámicas del entorno doméstico-familiar desde una mirada cuantitativa ha puesto de manifiesto la persistencia de las desigualdades de género en el reparto del trabajo doméstico y los cuidados. Un enfoque cualitativo, por su parte, saca a la luz la complejidad desde la que se constituyen dichas desigualdades, al tiempo que ofrece la oportunidad de abordar la variedad y los matices, así como las posibilidades de resignificación, tomando como eje el principio de reciprocidad.

El análisis cuantitativo muestra la persistencia de la división sexual del trabajo y las desigualdades de género que genera. Las mujeres continúan desempeñando la mayor parte del trabajo doméstico y de cuidados, independientemente de su edad y de su relación con el mercado laboral. Una participación cada vez mayor por parte de ellas en las ocupaciones asalariadas, implica que la carga global de trabajo se incline hacia sus espaldas, menguando su tiempo de ocio y vida social. Así, si bien producen la mayor parte de bienes y servicios necesarios para garantizar los niveles de bienestar y riqueza de la sociedad, invierten su tiempo en ocupaciones que, aunque resulten imprescindibles para la pervivencia, no gozan de reconocimiento y prestigio social. Una vez más, los datos sobre empleo del tiempo muestran que lo doméstico-familiar engloba un volumen importante de tiempo, que es desempeñado principalmente por las mujeres y que, asimismo, constituye el ámbito en el que las desigualdades de género son más acusadas. La desigual valoración y reconocimiento social de unos trabajos y otros crea jerarquías entre los tiempos dedicados al trabajo doméstico-familiar, al mercado y al ocio y vida social, dificultando el desarrollo de unas relaciones basadas en la equidad.

Un análisis más atento a los significados y las representaciones, sin embargo, saca a la luz la complejidad de las dinámicas del entorno doméstico-familiar y enriquece los resultados del estudio cuantitativo. En el nivel discursivo, se han identificado cuatro posiciones en torno a la relación entre las adscripciones de género y el trabajo doméstico y los cuidados que dependen de la posición social de quien construye el relato: identificación, problematización, reinvención y reafirmación. La (auto)asignación de los roles tradicionales de género, la moralización del tiempo y el desarrollo del principio de reciprocidad que rige la provisión de cuidados, se interpretan de forma diferente según la posición discursiva, 
si bien esta interpretación no se construye siempre de forma sólida y homogénea. Así, se pueden definir tres posturas diferentes con relación a la crisis de cuidados: en la primera, la crisis parece no percibirse; en la segunda, se contempla con reticencia; y en la tercera, se define como una oportunidad.

En primer lugar, se percibe que las mujeres mayores $y$ los hombres con empleo asumen y, en cierta forma, refuerzan la división sexual del trabajo. Las del primer grupo se han socializado en los roles más tradicionales de género, se ocupan y preocupan del ámbito domésticofamiliar de una forma tal, que este llega a ser el principal eje estructurador de su día a día tanto en la práctica como en el nivel simbólico. El principio de reciprocidad se acepta y se da por cumplido: ellas donan su tiempo y, a cambio, sienten la satisfacción del deber cumplido y recogen gratitud de parte de aquellas personas que reciben lo ofrecido. Los del segundo grupo parten de una posición colaboradora en el ámbito doméstico-familiar, pero en el nivel discursivo no producen un imaginario que abogue por la corresponsabilidad. Reproducen el modelo "hombre ganador de pan / mujer ama de casa", lo que supone la asimilación de un principio de reciprocidad basado en la inequidad: aportan menos de lo que reciben. La crisis de cuidados parece no afectar a estos dos grupos, apenas se deja notar. En el resto de los grupos, sin embargo, se puede constatar que, aunque de diferentes formas y con diversos matices, se genera un cuestionamiento de la norma.

En segundo lugar, entre las mujeres con empleo no cualificado y los hombres jubilados y prejubilados se observan los cambios característicos de la crisis de cuidados con reticencia. Entre ellas, cabe matizar, sin embargo, que no se produce un discurso unívoco: se genera una tensión intergeneracional. Las más mayores acusan a las más jóvenes de poner en tela de juicio el principio de reciprocidad por estar en edad de procrear y no tener descendencia. Las más jóvenes, aunque a nivel discursivo reproducen el modelo de maternidad intensiva, apelando al sentimiento de culpa que les produciría tener criaturas y no poder atenderlas por su dedicación al mercado laboral, con su comportamiento ponen en cuestión la norma social pues, si bien es el momento de restituir la deuda, se niegan a dar. Las más mayores, por su parte, abogan por mantener el principio de reciprocidad en los términos anteriores a la crisis. Los hombres jubilados y prejubilados verbalizan de forma abierta la ruptura de la lógica del don, sienten incertidumbre y desasosiego ante la nueva situación pues no contemplan una solución factible para su restablecimiento: en el pasado han ejercido de hijos y han obedecido a sus padres (recibir), han ejercido de padres y han cuidado de sus hijos/as (dar) y de sus padres ya mayores (devolver), y ahora que se acerca el momento en el que ellos mismos precisen cuidados, no vislumbran ninguna posibilidad operativa de restitución. Contemplan con cierta añoranza la estabilidad, seguridad y certeza de la época anterior a la crisis.

En tercer y último lugar, se puede afirmar que entre las amas de casa a tiempo completo y las mujeres profesionales, aunque en grados muy distintos, se atisba un cuestionamiento de los roles tradicionales de género. Frente a la escasa resonancia social que tiene su posición social actual, las amas de casa a tiempo completo sacan a la luz su agencia al reproducir una identidad renovada, que dota de reconocimiento tanto a lo que hacen como a lo que son. Si bien no cuestionan el principio de reciprocidad, en el que las mujeres donan su tiempo a cambio de gratitud, se percibe cierto tono sacrificial en la renuncia que supone el don. No obstante, en la falta de acuerdo en torno al futuro de sus hijas se presenta un atisbo de apertura hacia un cuestionamiento por parte de las mujeres de la asunción de la función doméstica: no todas quisieran que sus hijas fuesen también amas de casa. El cuestionamiento es mucho más explícito y general entre las mujeres profesionales. No sin esfuerzo ni contradicciones, dejan ver su agencia tanto en sus comportamientos como en el nivel discursivo, a través de las estrategias que desarrollan para lograr tiempo para sí y mediante las reivindicaciones a favor de unas relaciones de género más equitativas basadas en un principio de reciprocidad simétrico. En estos dos grupos la crisis de cuidados se contempla como una oportunidad para resignificar las relaciones entre géneros y, principalmente en el último, para apostar por una nueva organización social del trabajo doméstico y los cuidados.

\section{AGRADECIMIENTOS}

La tesis doctoral en la que se inspira el texto fue llevada a cabo gracias a una beca predoctoral del gobierno vasco. El trabajo de campo al que se hace referencia forma parte de la investigación "Lógicas temporales y desigualdades de género. Un análisis cualitativo sobre la distribución del tiempo de hombres y mujeres en la Comunidad Autónoma de Euskadi", desarrollada gracias a la Subvención a Proyectos de Investigación 2007 concedida por el Instituto Vasco de la Mujer-Emakunde (referencia: 24/2007PRO). Agradezco a Izaskun Artegui, Ramón Ramos y Cristina García Sainz, la atenta lectura de este texto, sin embargo, la responsabilidad última del mismo es mía. 
1. El artículo toma como punto de partida algunas reflexiones presentadas en la tesis doctoral inédita El tiempo donado en el ámbito doméstico-familiar. Estudio sobre el trabajo doméstico y los cuidados, defendida por la autora en julio de 2012 en la Universidad del País Vasco.

2. La propuesta de abordar en estudio del ámbito doméstico-familiar a partir de la noción de tiempo donado, se ha desarrollado con mayor profundidad en trabajos anteriores (Legarreta Iza, 2008; Legarreta Iza, 2009; Legarreta Iza, 2011; Legarreta Iza, 2013; Legarreta, 2014a).

3. Los datos de la EPT han sido cedidos a la autora por el Instituto Vasco de Estadística - Eustat con fines de investigación. La EPT se lleva a cabo en la Comunidad Autónoma del País Vasco de forma quinquenal desde 1993. El trabajo de campo de la última encuesta se ha desarrollado en 2013, pero los datos todavía no están disponibles. Por tanto, los últimos datos publicados por el Eustat son de 2008. Las características técnicas de la EPT se encuentran en la página web del Instituto Vasco de Estadística - Eustat (disponible en: http:// www.eustat.es/estadisticas/tema_173/ opt_0/tipo_7/ti_Uso_del_tiempo/temas.html\#axzz2ziHfNQfc Consultado el 23/04/2013).

4. Se han llevado a cabo nueve grupos de discusión en diversas localizaciones geográficas de la Comunidad Autónoma del País Vasco, teniendo en cuenta las siguientes variables: género, edad, relación con el mercado laboral y composición del hogar. Todos ellos han tenido lugar entre noviembre de 2007 y enero de 2008 y han sido moderados por la autora, investigadora responsable del estudio.

5. Entre las mujeres se crean desigualdades entre empleadas y empleadoras, pero también entre aquellas que pueden externalizar parte del trabajo doméstico y de cuidados, y las que no.

6. La reflexión aquí recogida en torno al tiempo donado ha sido extraída mayormente de la tesis doctoral de la autora citada con anterioridad y referenciada en la bibliografía. Parte de lo expuesto en este apartado, por tanto, proviene de dicho trabajo anterior. Las reflexiones posteriores en torno a cómo opera la crisis de cuidados en las relaciones desarrolladas en torno al tiempo donado en el ámbito doméstico-familiar, según las diferentes posiciones discursivas, son inéditas.

7. La teoría del don ha sido desarrollada por Marcel Mauss en su obra Ensayo sobre los dones. Razón y forma del cambio en las sociedades primitivas (1925), ha tenido una notable repercusión en las teorías sobre el intercambio y ha sido objeto de diferentes aplicaciones. Para elaborar este artículo se han utilizado las siguientes referencias: la reflexión de Laura Bimbi (1991) sobre la economía del don en el ámbito doméstico-familiar, el abordaje de Joan Bestard (1998) respecto a la tecnología de la procreación, los trabajos de David Casado Neira (2003) en torno a la donación de sangre y la aproximación de Elixabete Imaz (2009) a la maternidad desde el don. Asimismo, para profundizar en el principio de reciprocidad, han sido de gran utilidad las reflexiones desarrolladas por Alvin W. Gouldner (1979) a este respecto.

8. Parte del análisis de los datos expuestos en este apartado ha sido extraído de la tesis doctoral de la autora citada con anterioridad y referenciada en la bibliografía.

9. Las mujeres asumen al día una carga global de trabajo de 6 horas y $50 \mathrm{mi}$ nutos y los hombres de 5 horas y 38 minutos, lo que conlleva que ellas dispongan de menos tiempo para otras actividades, principalmente para el ocio y la vida social. Analizando los datos de forma longitudinal, se percibe que la desigual distribución de la carga global entre mujeres y hombres no ha variado considerablemente con el paso del tiempo: en 1998 y en 2003 disminuye sensiblemente la carga de las mujeres y aumenta la de los hombres, pero la tendencia se invierte en el año 2008 mostrando una distribución similar a la de 1993. Desde esta perspectiva, se observan mayores cambios con relación al tiempo de trabajo de las mujeres que con relación al de los hombres. Entre ellas aumenta el empleado para el trabajo retribuido, pero tal incremento no se corresponde con un aumento similar en la dedicación al trabajo doméstico-familiar por parte de ellos. Dicha tendencia está acompañada por la disminución del tiempo invertido por las mujeres en las actividades doméstico-familiares, principalmente con relación al trabajo doméstico, pues le dedican casi una hora menos en 2008 que en 1993 (4 horas y 16 minutos en 1993 y 3 horas y 25 minutos en 2008).

10. Una de las mujeres narra que, queriendo simultáneamente ejercer la maternidad de forma intensiva y tener un empleo que se corresponda con su formación, opta por el teletrabajo como estrategia para la conciliación. Describe cómo tal decisión conlleva unas jornadas laborales que no respetan los ritmos circadianos y que derivan en falta de sueño. La falta de sueño, asegura, le produce problemas de salud que desembocan en un trastorno hormonal y una menopausia precoz.

11. La lucha contra el sentimiento de culpa se percibe, por ejemplo, en las estrategias que desarrollan, no sin esfuerzo, las mujeres profesionales para lograr tiempo para sí, tiempo propio: reducción de la jornada laboral, externalización de parte del trabajo doméstico y de cuidados, y determinación a la hora de decidir a qué se quiere dedicar el tiempo de libre disposición (Legarreta, 2014a). Las estrategias, en este caso, se entienden en el sentido propuesto por Michel de Certeau (1993/2001, pp. 391-425) en tanto que hacen referencia a una apropiación (de tiempo, y no tanto de espacio) que llega a cuestionar la relación de fuerzas existente en torno a las adscripciones de género.

12. La postura de las mujeres mayores es bien distinta: la vulnerabilidad se asume como característica intrínseca de las personas y del paso del tiempo, la vejez y la muerte se aceptan y se naturalizan, si bien se pone de manifiesto que el proceso de naturalización es también un aprendizaje. Sus narraciones resultan sumamente sugerentes pues, en cierta medida, se acercan a las propuestas que se están desarrollando en el marco de una ética del cuidado feminista (Molinier, 2011; Paperman, 2011). Se profundiza sobre este aspecto en un trabajo anterior (Legarreta Iza, 2014b). 


\section{BIBLIOGRAFÍA}

Adam, B. (1995). Timewach. The social analysis of time. Cambridge: Polity Press.

Adam, B. (1999). Cuando el tiempo es dinero. Racionalidades de tiempo conflictivas y desafíos a la teoría y la práctica del trabajo. Sociología del trabajo, 37, pp. 5-39.

Anderson, B. (2000). Doing the Dirty Work? The Global Politics of Domestic Labour. London, New York: Zed Books.

Arpal, J. (1997). Regularidades temporales y vida cotidiana. En Departamento de Justicia, Economía, Trabajo y Seguridad Social (ed.). Empleo y tiempo de trabajo: el reto de fin de siglo. Vitoria-Gasteiz: Gobierno Vasco.

Bacigalupe de la Hera, A. y Martín Roncero, U. (2007). Desigualdades sociales en la salud en la población de la Comunidad Autónoma del País Vasco. La clase social y el género como determinantes de la salud. Vitoria-Gasteiz: Ararteko.

Bestard, J. (1998). El lenguaje de la donación de material genético. En: Parentesco y modernidad. Barcelona: Paidós, pp. 224-228.

Bettio, F., Simonazzi, A y Villa, P. (2006). Change in care regimes and female migration: the 'care drain' in the Mediterranean. Journal of European Social Policy, 16, 3, pp. 271-285. https://doi. org/10.1177/0958928706065598

Bimbi, L. (1991). L'economia del dono. En Balbo, L. (ed.) Tempi di vita. Studi e proposte per cambiarli. Milán: Giangiacomo Feltrinelli, pp. 62-68.

Casado Neira, D. (2003). La teoría clásica del don y la donación de sangre. Revista Internacional de Sociología, Tercera Época, 34, pp. 107-133. https://doi. org/10.3989/ris.2003.i34.281

Certeau, M. de (1993/2001). La invención de lo cotidiano (vol. 1: Artes de hacer). México: Universidad Iberoamericana.

Cordoni, E. (1993). Las mujeres cambian los tiempos. Cuadernos de Relaciones Laborales, 2, pp. 221-237.

Díaz Gorfinkiel, M. (2008). El mercado de trabajo de los cuidados y la creación de las cadenas globales de cuidado: ¿cómo concilian las cuidadoras? Cuadernos de Relaciones Laborales, 26, 2, pp. 71-89.

Ehrenreich, B. y Hochschild, A. R. (eds.) (2002). Global Woman. Nannies, Maids and Sex Workers in the New Economy. New York: Owl Books.

Esquivel, V. (2011). La "economía del cuidado": un recorrido conceptual. En: Sanchís, N. (comp.). Aportes al debate del desarrollo en América Latina: una perspectiva feminista. Buenos Aires: Red de Género y Comercio-América Latina, pp. 20-30. [En línea] Disponible en: http:// www.remte-bolivia.org/attachments/ article/133/Aportes\%20al\%20debate $\% 20$ del\%20desarrollo\%20en\%20 America\%20Latina.pdf\#page $=20$

Gouldner, A. M. (1979). Reciprocidad y autonomía en la teoría funcionalista. La norma de la reciprocidad: formulación preliminar y la importancia de dar algo por nada. En: La sociología actual: renovación y crítica. Madrid: Alianza, pp. 182-281.

Héritier-Augé F. (1985) La Cuisse de Jupiter. Réflexions sur les nouveaux modes de procréation. L'Homme, XXV, 94, pp. 5-22.

Hochschild, A. R. (1995). The Culture of Politics: Traditional, Post-modern, Coldmodern, and Warm-modern Ideals of Care. Social Politics, 2, 3, pp. 331-346. https://doi.org/10.1093/sp/2.3.331

Hochschild, A. R. (2001). Las cadenas mundiales de afecto y asistencia y la plusvalía emocional. En: Giddens, A. y Hutton, W. (eds.). En el límite. La vida en el capitalismo global. Barcelona: Tusquets.

Imaz Martínez. E. (2009). Mujeres gestantes, madres en gestación. Representaciones, modelos y experiencias en el tránsito a la maternidad de las mujeres vascas contemporáneas. Leioa: Servicio Editorial de la Universidad del País Vasco.

Legarreta Iza, M. (2007). La globalización del trabajo doméstico y de cuidados. Nuevas configuraciones de la domesticidad. En Arpal, J. y Mendiola, I. (eds.). Estudios sobre cuerpo, tecnología y cultura. Leioa: Servicio Editorial de la Universidad del País Vasco.

Legarreta Iza, M. (2008). El tiempo donado en el ámbito doméstico. Reflexiones para el análisis del trabajo doméstico y los cuidados. Cuadernos de Relaciones Laborales, 26, 2, pp. 45-69.

Legarreta Iza, M. (2009). Le temps donné dans travail domestique et de care. Multitudes, 37-38, pp. 106-112. https:// doi.org/10.3917/mult.037.0106
Legarreta Iza, M. (2011). Lógicas temporales y desigualdades de género: el tiempo donado en el ámbito doméstico. En Chivite de León, M. J., Hernández Pérez, M. B. y Monzón, M. E. (eds.). Frontera y género. Madrid: Plaza y Valdés, pp. 237-247.

Legarreta, M. (2012). El tiempo donado en el ámbito doméstico-familiar. Estudio sobre el trabajo doméstico y los cuidados. [Tesis doctoral inédita]. Universidad del País Vasco-Euskal Herriko Unibertsitatea: Bilbao.

Legarreta Iza, M. (2013). El tiempo como herramienta para la economía feminista. (Una propuesta a partir del estudio del trabajo doméstico y los cuidados). Comunicación presentada a las IV Jornadas de Economía Feminista, Sevilla, del 3 al 5 de octubre. [En línea]. Disponible en: http:// riemann.upo.es/personal-wp/congresoeconomia-feminista/files/2013/10/Legarreta-Matxalen.pdf.

Legarreta, M. (2014a). La crisis de cuidados y sus rasgos temporales: tiempo encarnado, tiempo moralizado y tiempo politizado. En: Araújo, E., Duque, E., Franch, M. y Durán, J. (eds). Tempos Sociais e o Mundo Contemporâneo. As crises, As Fases e as Ruturas. Braga: Universidade do Minho, pp. 21-33.

Legarreta Iza, M. (2014b). Cuidados y sostenibilidad de la vida: una reflexión a partir de las políticas de tiempo. Papeles del CEIC, 104, pp. 1-36. https://doi. org/10.1387/pceic. 12427

Lewis, J. (2007/2011). Género, envejecimiento y el 'nuevo pacto social': la importancia de desarrollar un enfoque holístico de las políticas de cuidados. En: Carrasco, C., Borderías, C. y Torns, T. (eds.). El trabajo de cuidados. Historia, teoría y políticas. Madrid: Catarata, pp. 336-358.

Lewis, D. J. y Weigert A. J. (1981/1992). Estructura y significado del tiempo social. En: Ramos Torre, R. (comp.). Tiempo y sociedad. Madrid: CIS.

Mauss, M. (1925/1971). Ensayo sobre los dones. Razón y forma del cambio en las sociedades primitivas. En: Mauss, M. Sociología y antropología. Madrid: Tecnos.

Menzies, H. (2000). Cyberspace, Time and Infertility: Thoughts on Social Time and the Environment. Time \& Society, 9, 1, pp. 75-89. https://doi. org/10.1177/0961463X00009001005 
Molinier, P. (2011). Antes que todo, el cuidado es un trabajo. En: Arango Gaviria, L. G. y Molinier, P. (comps.). El trabajo y la ética del cuidado. Medellín: Universidad Nacional de Colombia / La Carreta, pp. 45-64.

Murillo, S. (1995). Espacio doméstico: el uso del tiempo. En: Tobío Soler, C. y Denche, C. (eds.). El espacio según el género. ¿Un uso diferencial? Madrid: Universidad Carlos III de Madrid, pp: 133-140.

Murillo de la Vega, S. (1996). El mito de la vida privada. De la entrega al tiempo propio. Madrid: Siglo XXI.

Orozco, A. (2010). Diagnóstico de la crisis y respuestas desde la economía feminista. Revista de Economía Crítica, 9, pp. 131-144.

Orozco, A. (2011). De vidas vivibles y producción imposible. [En línea]. Disponible en: http://www.rebelion.org/noticia. $p h p$ ?id=144215

Paperman, P. (2011). La perspectiva del care: de la ética a lo político. En: Arango
Gaviria, L. G. y Molinier, P. (comps.) El trabajo y la ética del cuidado. Medellín Universidad Nacional de Colombia / La Carreta, pp. 25-44.

Parella Rubio, S. (2003). Mujer, inmigrante y trabajadora: la triple discriminación. Barcelona: Anthropos.

Ramos Torre, R. (1990). Cronos dividido: uso del tiempo y desigualdad entre mujeres y hombres en España. Madrid: Instituto de la Mujer.

Ramos Torre, R. (2006). La situación general del empleo del tiempo en la C. A. de Euskadi: estructura, dinámica y comparación. En: Encuesta de Presupuestos de Tiempo. 2003. Monográficos. VitoriaGasteiz: Eustat. Instituto Vasco de Estadística, pp. 18-49.

Ramos Torre, R. (2007). Metáforas sociales del tiempo en España: una investigación empírica. En: Prieto Rodríguez, C. (ed.). Trabajo, género y tiempo social. Madrid: Hacer editorial / Universidad Complutense, pp. 173-204.
Ramos Torre, R. (2009). Metáforas del tiempo en la vida cotidiana: una aproximación sociológica. Acta Sociológica, 49, pp. 51-69.

Ramos Torre, R. (2011). Más allá de las cifras: la dimensión teórica y cualitativa del tiempo. En: Durán Heras, M. A. (dir.) El trabajo de cuidado en América Latina y España. Madrid: Fundación Carolina, pp. 75-87.

Salazar Parreñas, R. (2001). Servants of globalization. Women, migration, and domestic work. Stanford: Stanford University Press

VV.AA. (2004). Hogares, cuidados y fronteras: derechos de las mujeres inmigrantes y conciliación. Madrid: Traficantes de Sueños.

Zimmerman, M. K. (2006). Globalization and multiple crises of care. En: Zimmerman, M. K, Litt, J. S. y Bose, C. E. Global dimensions of gender and carework. Stanford University Press, pp. 9-27. 